\title{
Dossier documental sobre comunicación alternativa y aumentativa
}

\author{
CARMe Rosell \\ Funcación L'Espiga
}

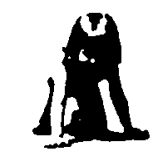

\section{Resumen}

Este dossier proporciona, sin ánimo de exbaustividad, la información que nos ba parecido más relevante para los profesionales de España que tratan con personas que necesitan usar técnicas alternativas y aumentativas de comunicaciôn. En él se incluye una bibliografía comentada y reseñas de diferentes revistas periódicas, de producciones audiovisuales y de ayudas técnicas para la comunicación. También se incluye una relación de entidades que pueden proporionar información sobre el tema y una lista de direcciones de casas comerciales que fabrican y/o distribuyen ayudas técnicas para la comunicación.

Palabras clave: Documentación bibliográfic a; Ayudas técnicas para la comunicación; Entidades e instituciones; Fabricantes y distribuidores.

\section{Dossier on Alternative and Augmentative Communication}

\section{Abstract}

The present documentation, though not exhaustive, is directed at professionals working in Spain in Augmentative and Alternative Communication. It summarizes the most relevant information in this field, including a commented bibliograpby and reviews of different periodicals, audiovisual productions, and technical aids for communication. It also includes a list of institutions that can provide information on this topic, and addresses of firms manufacturing and/or distributing technical aids for communication.

Key words: Documentation; Ternbical aids for communication; Institutions; Manufacturers and distributors. 


\section{BIBLIOGRAFIA COMENTADA}

En este comentario bibliográfico nos proponemos realizar un resumen de los principales libros y documentos publicados, preferentemente, en nuestro país, con la sola excepción del libro de Sara Blackstone, que se ha considerado indispensable incluir, a pesar de no haber sido traducido del inglés.

Para una reseña más amplia de las diferentes obras que aquí comentamos se puede consultar el documento de Basil, Soro y Rosell (1992): Bibliografias comentadas. La comunicación en alumnos con deficiencia motora. Madrid: M.E.C. y C.N.R.E.E.

Basil, C,; Ruiz, R. (1988). Sistemes de comunicació no vocal per a nens amb, disminucions físiques (2.a edición). Barcelona: Generalitat de Catalunya. Documents d'Educació Especial 3. Traducido al castellano: 1985. Sistemas de comunicación no vocal para niños con disminuciones físicas. Madrid: Los libros de Fundesco.

La obra constituye una introducción a los sistemas alternativos de comunicación, especialmente útil para los profesionales relacionados con la evaluación e intervención educativa de personas con discapacidad motora.

Presenta los aspectos más relevantes sobre las características de los sistemas alternativos de comunicación, los criterios para la evaluación y los procesos de enseñanza de dichos sistemas. Los principales temas tratados en diferentes capítulos son: Los objetivos de los sistemas aumentativos de comunicación y la población susceptible de utilizarlos, tipos de signos gráficos, formas de indicación y ayudas técnicas, criterios de selección del primer vocabulario, criterios de elección de los candidatos y selección del sistema de signos. El último capítulo trata sobre la utilidad de los sistemas alternativos y su papel en el desarrollo cognitivo y del habla.

Destacar la bibliografía específica para cada uno de los contenidos incluida al final de los respectivos capítulos, útil para que la persona interesada en el tema pueda ampliar información y especializarse en el mismo.

Basil, C,; Puig de la Bellacasa, $\boldsymbol{R}$ (Eds.) (1990). Comunicación aumentativa. Curso sobre sistemas y ayudas técnicas de comunicación no vocal (2." edición). Madrid: INSERSO.

Texto revisado de las conferencias desarrolladas en el curso de «comunicación no vocal» celebrado en Madrid en 1985. Los capítulos, de diferentes autores, constituyen una introducción y aportación práctica sobre la comunicación alternativa, de especial interés para estudiantes y profesionales interesados en el desarrollo de la comunicación.

Carme Basil, resume la clasificación y expone conceptos referidos a la terminología y papel de los sistemas de signos gráficos y manuales. Ramón Puig de la Bellacasa y Paloma Sánchez de Muniaín resumen las aportaciones tecnológicas desarrolladas en el ámbito de la rehabilitación. Robert Ruiz, en el tercer capítulo, trata el tema del proceso de toma de decisiones y la valoración psicopedagógica para el uso de los sistemas alternativos de comunicación. Rosario Peláez trata de los sistemas de signos gráficos SPC y PICSYMS. Carme Basil expone los obstáculos para la comunicación con los que puede encontrarse la persona con discapacidad motora y las consecuencias en su desarrollo. Javier Tamarit trata del papel de los sistemas aumentativos para mejorar la comunicación en poblaciones con deficiencia mental, autismo, psicosis infantil, etc. Marian Ochoa de Eribe ilustra el proceso educativo para la comunicación seguido con niños que utilizan el sistema Bliss, incluye un apartado sobre el desarrollo del lenguaje escrito. Pilar Such trata algunos aspectos teórico-prácticos sobre el sistema Bliss. En el último capítulo, Ramón Puig de la Bellacasa describe los recursos y organización de la comunicación aumentativa en diferentes países con experiencia en el tema y termina con una descripción de la situación en España. 
Blackstone, S. W. (Ed.) (1986). Augmentative Communication: An introduction. Rockville: American Speech Language Hearing Association.

Constituye una exhaustiva revisión de materiales, procedimientos, experiencias, etc., disponibles en Comunicación Aumentativa.

Adecuado para estudiantes de cursos avanzados sobre comunicación aumentativa y apropiado como libro de consulta para profesionales con experiencia en el tema.

Los diferentes capítulos tienen como autores a personas de reconocido prestigio internacional en este campo. El primer capítulo, de introducción, está elaborado por G. C. Vanderheiden y D. E. Yoder. El segundo capítulo, de H. C. Shane, trata de las metas y usos de la comunicación aumentativa. El tercer capítulo, de G. C. Vanderheiden, versa sobre los sistemas de comunicación y sus componentes, con una descripción detallada de las características de los diferentes sistemas y de la tecnología en uso. El capítulo cuarto, a cargo de K. M. Yorkston y G. Karlan, trata sobre los procedimientos de evaluación, definiendo la evaluación como un proceso de identificación de los problemas comunicativos, encaminado a la elaboración de un plan sistemático de intervención. El quinto capítulo, a cargo de A. W. Kraat, trata de la definición de objetivos para la intervención. La autora enfatiza la necesidad de que la intervención garantice una mejora real de las habilidades de interacción y comunicación del usuario con las demás personas, y proporciona datos para lograrlo. El capítulo sexto, estrategias de intervención, consta de una parte introductoria elaborada por S. Blakstone y de una compilación de pequeños trabajos de autores diversos. El capítulo séptimo trata sobre la evaluación de la eficacia de los programas de intervención, a cargo de D. R. Beukelman. El capítulo octavo, de C. G. Cohen, hace referencia a los servicios de habilitación total a lo largo de la vida. El noveno y último capítulo, a cargo de P. Mathy-Laikko y D. E. Yoder, delimita las necesidades y directrices para el futuro de la comunicación aumentativa.

Fernández de Villalta, $\boldsymbol{M}$. (Ed.) (1988). Tecnologias de la información y discapacidad. Madrid: Los libros de Fundesco.

En esta obra se describen los distintos tipos de discapacidad que pueden hacer necesario el uso de las nuevas tecnologías como ayudas técnicas, se hace referencia a los problemas de visión, de audición, de habla, de motricidad e intelectuales, exponiéndose los recursos y adaptaciones disponibles para cada una de las necesidades. Asimismo, se definen y describen las nuevas tecnologías de la información. En primer lugar, se realiza una exposición de los logros alcanzados a nivel internacional, para pasar a exp!icar la situación y perspectivas de futuro en el estado español. Los diferentes aspectos han sido tratados por personas con experiencia directa en el tema. Por último destacar que la obra aporta información sobre centros, asociaciones y proyectos de investigación relacionados con el tema, así como definiciones de términos técnicos y bibliografía.

Gardeazábal, L. (Ed.) (1989). Aplicaciones del ordenador y de las nuevas tecnologias en la ayuda a personas con discapacidad. San Sebastián: Servicio Editorial Universidad del País Vasco.

Recopilación de las ponencias del CVII curso de verano celebrado en San Sebastián. El objetivo del curso era presentar, a profesionales especializados en el tema, la contribución que las nuevas tecnologías pueden aportar a las personas con grave discapacidad motora.

Ramón Puig de la Bellacasa comenta la cotidianidad de las ayudas técnicas y su papel en la mejora de la calidad de vida de las personas en general y de la persona con discapacidad en particular. Resalta la necesidad de investigación en nuestro país. Jan Ingvar Lindstrom presenta un capítulo en inglés sobre terminales telemáticos para 
personas con discapacidad. También en inglés aparece la ponencia presentada por Luis Azevedo, que trata sobre la tecnología de la rehabilitación y el papel de la universidad en este campo. Aguilera y colaboradores exponen, en el capítulo titulado Aplicaciones del procesamiento del habla en la ayuda a personas con discapacidad, sus investigaciones dedicadas a personas con problemas de visión y audición. Alicia Casals, en el capítulo titulado Aplicaciones del procesado de imágenes en las ayudas a personas con discapacidad, aborda el tema de captura, procesamiento y transmisión de imágenes para invidentes. Por último, Agustín Arruabarrena y Julio González dedican un capítulo a los dispositivos de comunicación, exponiendo las características de los comunicadores y la necesidad de que respondan a las necesidades de los usuarios.

Musselwbite, C. R. (1990). Juegos adaptados para niños con necesidades educativas especiales. Madrid: INSERSO.

Dirigido especialmente a los diferentes profesionales relacionados con niños con discapacidad: educadores especializados, logopedas, terapeutas ocupacionales, fisioterapeutas, etcétera.

En el libro podemos encontrar ideas, procedimientos, adaptaciones, material, etc., para conseguir que el niño con discapacidad pueda jugar de una forma realmente activa y adaptada a sus intereses y necesidades y, mediante el juego, aprender pautas básicas de comunicación.

La autora realiza una descripción del desarrollo de las técnicas de juego en niños con desarrollo normal, expone estrategias utilizadas de forma natural por los adultos, añadiendo de qué forma y con qué frecuencia es necesario utilizarlas en niños con necesidades especiales. Expone criterios de selección y adaptación de los materiales de juego según las necesidades del niño. Aporta ideas para incorporar el juego adaptado a la instrucción relacionada con las áreas de seguimiento visual y toma de decisiones. Por último, sugiere sistemas de apoyo para el juego adaptado, como la creación de ludotecas, soporte a los padres y cuidadores, etcéterạ.

En resumen, el libro intenta ser un compendio sobre el juego adaptado con numerosas citas a otros autores, incluyendo referencias a escalas de evaluación y materiales.

Peña,J. (1992, 2. ${ }^{a}$ Ed. Revisada). Manual de logopedia. Barcelona: Masson, S.A.

Libro de consulta para estudiantes y profesionales implicados en la educación de personas con trastornos de lenguaje y comunicación. En los diversos, capítulos, escritos por diferentes autores, se plantean definiciones, conceptos teóricos y propuestas relacionadas con la evaluación y aplicación de procedimientos de intervención. A destacar los capítulos especialmente relacionados con la intervención educativa del habla en personas con discapacidad motora: Bagunyà y Sangorrín dedican un capítulo a las disartrias, Puyuelo expone los principales puntos a tener en cuenta en la intervención logopédica en personas con parálisis cerebral, Monfort expone los trastornos del aprendizaje del lenguaje escrito y Carme Basil, en un capítulo original para esta segunda edición, presenta una introducción a los Sistemas Alternativos y Aumentativos de Comunicación.

Soro, $\boldsymbol{E}$; Marco, $\boldsymbol{F}$. (1990). Sistemas aumentativos de comunicación. Tema cinco: Las necesidades educativas especiales del niño con deficiencia motora. Serie: Formación. Madrid: M.E.C. y C.N.R.E.E.

Este trabajo forma parte de una publicación que con diferentes trabajos de varios autores aborda distintos aspectos de las necesidades educativas del niño con deficiencia motora. Va dirigido a profesionales directamente relacionados con la educación de niños con dificultades motoras pero con habilidades intelectuales suficientes como para poder acceder al currículo escolar ordinario. 
En este documento se sugieren soluciones prácticas a los problemas planteados por la integración de alumnos con dificultades motoras y usuarios de sistemas aumentativos en los centros ordinarios y se apunta la necesidad de introducir adaptaciones, ayudas técnicas, etc., para facilitar las diferentes actividades escolares, de forma que su uso no comporte dificultades para la integración. Aporta alternativas para que escolares con dificultades en el ámbito de la comunicación y el lenguaje puedan acceder al currículo ordinario. Dedica un capítulo al proceso de toma de decisiones y evaluación para el uso de sistemas aumentativos de comunicación mediante matrices de toma de decisiones. Por último, trata sobre cuestiones de organización escolar, enfatizando la necesidad de planificar el trabajo de forma coordinada entre los diferentes profesionales.

Cabe destacar la aportación de información sobre otros materiales de consulta y de bibliografía sobre el tema preferentemente en lengua castellana.

von Tetzchner, S. y Martinsen, $\boldsymbol{H}$. (1993). Introducción a la enseñanza de signos y al uso de ayudas técnicas para la comunicación. Madrid: Visor.

Se trata de una obra eminentemente práctica, dirigida tanto a profesionales que ya trabajan en el tema como a estudiantes y lectores con poca experiencia.

El autor defiende que todas las personas que no pueden usar el habla en la comunicación con los demás necesitan un sistema de comunicación alternativo, que cumplirá funciones distintas según el nivel y condiciones para adquirir la comprensión y el uso del habla. Argumenta que la enseñanza debe empezar sin demora, sin necesidad de que existan prerrequisitos para el aprendizaje de la comunicación, en todos aquellos niños que no hablan a la edad habitual, aunque presenten retrasos muy profundos. Aporta estrategias concretas y directamente aplicables para iniciar la enseñanza de la comunicación y del lenguaje dirigida a personas muy afectadas y también a usuarios con discapacidad motora grave pero con buen potencial intelectual.

A lo largo de la exposición pueden encontrarse constantes ejemplos reales de aplicación. Asimismo aparecen ilustrados más de cien signos manuales y más de doscientos signos gráficos que han sido adaptados para la edición española del original noruego. Por último destacar que los autores proporcionan un listado de estudios de casos, especificando las características de las personas implicadas en el aprendizaje y de los sistemas y técnicas usados.

\section{REVISTAS}

En nuestro país no existe, en la actualidad, ninguna revista especializada en el tema de Comunicación Aumentativa y Alternativa, por lo que reseñaremos las revistas españolas en las que suelen presentarse artículos sobre el tema, así como alguna revista internacional especializada en el mismo.

AAC. Augmentative and Alternative Communication. One James Street South, P.O. Box 620, L.C.D. 1, Hamilton, Ontario L8N 3K7, Canadá. Tel: 416 522-7017. Fax: $416522-7839$.

Revista oficial de la Sociedad Internacional para la Comunicación Aumentativa y Alternativa (ISAAC). En ella aparecen trabajos relacionados con el desarrollo de sistemas y tecnología y, especialmente, trabajos relacionados con la evaluación, tratamiento, habilitación y educación de personas que necesitan usar estas técnicas.

CLOSING THE GAP. Route 2, P.0. Box 68 Henderson, 56044 Minessota, EE.UU. 
Revista sobre ordenadores, hardware y software, y discapacidad. Con reseña de los materiales existentes en el mercado informático adecuados para personas con discapacidad.

COMMUNICATION OUTLOOK. Artificial Language Laboratory, Computer, Science Department, Michigan State University, East Lansing, Michigan 48824, EE.UU.

Tel: 517 353-0878.

Revista de información sobre tecnología, aplicaciones de Sistemas Aumentativos de Comunicación, experiencias personales de usuarios, incluye aplicaciones del ordenador.

INFODIDAC, Revista de Informática y Didáctica. Puenteareas, 15, 28002 MADRID. Tel: (91) 41361 03, Fax: (91) 4027348.

Publicación que aporta las novedades de la informática en el ámbito de la educación y la formación.

REVISTA DE LOGOPEDIA, FONIATRIA Y AUDIOLOGIA. Organo Oficial de la A.E.L.F.A. Balmes, 151, pral. 08008 BARCELONA. Tel: (93) 217 9954.

Pueden encontrarse trabajos sobre tratamiento y enseñanza de comunicación y lenguaje oral en diferentes poblaciones y ámbitos. También pueden encontrase trabajos sobre habilitación del lenguaje oral en personas con discapacidad motora y, esporádicamente, sobre comunicación alternativa y aumentativa.

\section{PRODUCCIONES AUDIOVISUAIES}

En el mercado internacional podemos encontrar gran diversidad de vídeos, de divulgación general o para profesionales especializados en el tema de la comunicación aumentativa. En nuestro país ha sido recientemente publicado en idioma casteIlano, catalán e inglés el siguiente vídeo:

Basil, C.; Soro, E.; VonTetzchner, S. (1992). Estrategias iniciales para la enseñanza de comunicación aumentativa. ICE Institut de Ciencies de l'Educació. Universitat de Barcelona. Carrer dels Angels, 18. 08001 Barcelona. Teléfono: (93) 31832 61. Fax: (93) 3024583.

Vídeo realizado como apoyo didáctico para los cursos sobre Comunicación Aumentativa y Alternativa dirigidos a estudiantes universitarios, así como a profesionales que tratan con niños que necesitan aprender signos y/o usar ayudas técnicas para la comunicación.

Se ilustran diversas estrategias para la enseñanza de signos manuales y gráficos, dirigidas a personas con graves problemas para la expresión y la comprensión del lenguaje.

\section{AYUDAS TECNICAS}

Denominamos ayudas técnicas para la comunicación a una serie de instrumentos destinados a facilitar la comunicación de aquellas personas que tienen graves dificultades de producción del habla oral. Pueden dividirse en diferentes categorías dependiendo de su grado de complejidad, desde sencillas libretas de comunicación a ordenadores con programas de comunicación dotados de voz sintetizada, altamente sofisticados. Seguidamente expondremos las principales categorías de instrumentos, sin pretender ser exhaustivos, ya que debido al continuo avance de la tecnología en 
este campo, el repertorio de instrumentos y casas comerciales a nivel internacional es muy amplio. En este escrito nos remitiremos a proporcionar la información más contrastada en nuestra experiencia diaria. La relación de direcciones de las casas comerciales citadas en el escrito se realiza en el apartado especialmente dedicado a ello.

\section{Comunicadores}

Se trata de instrumentos alimentados por baterías, lo que permite un funcionamiento autónomo durante un número variable de horas. Algunos de ellos, pocos en la actualidad, deben ir conectados a la red eléctrica.

Las formas de indicación de los mensajes comunicativos son diversas:

- Directamente con una parte del cuerpo o instrumentos acoplados (cabezales licornio, indicadores luminosos, etc.) a una superficie sensible, donde están situados los signos gráficos seleccionados.

- Mediante conmutadores. Los elementos comunicativos se seleccionan por barrido.

Las formas más habituales son:

a) Aguja de reloj. Mientras el usuario mantiene apretado un conmutador, la aguja se desplaza, cuando deja de hacerlo se mantiene fija indicando el símbolo. Puede acoplarse un temporizador, de forma que cada vez que presiona el conmutador, la aguja se desplaza un espacio predeterminado. Algunos de ellos son metálicos y permiten fijar los signos gráficos con imanes, otros son de metacrilato, pesan muy poco y son fácilmente portables en silla de ruedas, aunque hoy en día se usan poco para la comunicación por carecer de salida en voz. En cambio, dada la facilidad de colocación de los símbolos, son muy utilizados para realizar actividades escolares por parte de niños con dificultades de indicación directa. Diferentes distribuidores son: Don Johnston, Quest Educational Designs, Tash.

b) Teclas o casillas con señalización luminosa. Cada casilla donde se coloca un elemento comunicativo consta de una pequeña luz que el usuario debe iluminar para indicar, mediante uno o varios conmutadores. Las formas de barrido son diversas, valorándose la rapidez de indicación. Este tipo de comunicadores tienen diferentes formatos y capacidad de elementos comunicativos (desde uno a infinidad, si están dotados de voz sintetizada), pudiendo preprogramarse previamente dependiendo de las necesidades del usuario. Son distribuidores, entre otros: Canon, Liberator Ltd., Mardis, Possum, Prentke Romich Company, Quest Educational Designs, Zygo.

c) Barrido auditivo. Pueden grabarse varios mensajes comunicativos en las diferentes casillas. Al seleccionar una determinada casilla, directamente o mediante conmutadores, el usuario oye los mensajes y selecciona el adecuado. Es especialmente indicado para personas con dificultades visuales. Algunos distribuidores son: Zygo, AbleNer, etcétera.

En el momento de la selección del instrumento es importante valorar:

- la portabilidad: tamaño, peso y dotación de un aparato especialmente diseñado para colocar el instrumento en la silla de ruedas;

- que el comunicador esté dotado de salida de voz, lo que facilita y normaliza en alto grado la interacción comunicativa. Los tipos de voz más utilizados son la voz digitalizada (el aparato dispone de una memoria variable que permite grabar un repertorio limitado de mensajes comunicativos) y la voz sintetizada o de «texto a habla» (las posibilidades de construcción de enunciados puede ser tan amplia como el habla oral sin ayuda). En los comunicadores con voz sinterizada el usuario suele 
poder seleccionar signos gráficos de forma global o deletrear los mensajes, en este último caso las posibilidades comunicativas son ilimitadas pero requiere que el usuario sepa escribir. No existen en la actualidad comunicadores dotados de voz sintetizada en ninguno de los idiomas del estado español, algunas casas comerciales extranjeras están trabajando en el tema, especialmente Prenke Romich Company. Sí existen, en cambio, algunos comunicadores a los que se puede acoplar un sinterizador de voz portátil en castellano, como el comunicador CANON (versión reciente), que funciona con el sintetizador APOLO, o los comunicadores POLYCOM y otros que funcionan con INFOVOX (ver más abajo, el apartado sobre sintetizadores de voz). Son distribuidores: Canon, Multitec, etcétera.

\section{Periféricos para el ordenador}

Cuando se trata de considerar un ordenador para personas con discapacidades hay que tener en cuenta una serie de características: nitidez de los signos gráficos en pantalla, posibilidad de adaptación de diversos periféricos accesorios (conmutadores, teclados alternativos, connectabilidad a una pantalla en color o más grande, etcétera).

También es especialmente indicado utilizar materiales del mercado ordinario que pueden facilitar el correcto uso del ordenador, como un filtro polarizador para la pantalla del ordenador, soporte móvil para el monitor, discos de tamaño $31 / 2$, protector de teclado contra la humedad, polvo, etcétera.

Existen periféricos para el ordenador especialmente diseñados para personas con discapacidad motora, los más utilizados se detallan a continuación:

Ratones adaptados. Permiten realizar las mismas funciones que el ratón convencional a personas con menos control manual, o que utilizan otras partes del cuerpo (cabeza, pies, etc.). Algunos de ellos son:

- Head-Master. Se acopla a un cabezal y permite controlar el ratón con ligeros movimientos de la cabeza. El clic puede realizarse mediante un conmutador accionado con el pie, la mano, por soplo, etc. Distribuido por Personic Corparation, Liberator Ltd, Pointer Systems, etcétera.

- Trackball (ratón al revés). Se controla moviendo con los dedos, mano, brazo, pie, etc., una bola de tamaño superior al normal, mientras el resto del ratón permanece inmóvil. Disponible en las diferentes casas comerciales de ordenadores.

- Multimouse. Consiste en un interface al que pueden conectarse diversos dispositivos de control: un juego de pedales de direcciones, un joystick anatómico estándar, un juego de teclas de direcciones de gran tamaño, etc. Distribuido por Don Johnston.

Cobertor de teclado. Tablero, generalmente de metacrilato, con agujeros correspondientes a las teclas. Se coloca encima del teclado convencional. Debe adaptarse a los teclados de los diferentes modelos de ordenador ya que la posición de teclas suele variar ligeramente. Distribuido por Don Johnson.

Teclados adaptados. Existen diversos teclados, alternativos al convencional, que permiten su utilización por parte de personas con diferentes características y posibilidades motrices. Algunos de ellos son:

- teclado ampliado. Las teclas son más grandes y están más separadas que las convencionales, su tamaño puede variar según los modelos. Es adecuado para aquellas personas con movimientos poco precisos. Distribuido por Don Johnston, Fondation Suisse pour les Téletheses, Possum, etc. 
- teclado reducido. Las teclas son más pequeñas y juntas que en el teclado convencional. Algunos deben activarse mediante un punzón. Adecuados para personas con falta de tonicidad muscular y dificultad en realizar movimientos amplios pero con buena coordinación de movimientos en un espacio pequeño. Existen diferentes modelos en las diversas casas comerciales: Don Johnston, Fondation Suisse pour les Téletheses.

- tablero sensible. Tablero liso con áreas sensibles que se activan mediante presión. Estas áreas pueden definirse y asociar una determinada función a cada casilla o definir una área de varias casillas de forma que la superficie destinada a una misma función sea grande, adaptándose a las habilidades visuales, motoras y cognitivas de los usuarios. Una vez definido el tablero, debe colocarse encima una plantilla con las diferentes funciones. Algunas casas comerciales: AB Micro Express, Adaptive Communication Systems, Don Johnston, etcétera.

Emuladores de teclado. Consisten en un software y un hardware que hay que instalar en el ordenador. Permiten periféricos de entrada alternativos: conmutadores para utilizar el ordenador por barrido o con morse, teclados ampliados o reducidos, etc. Existe el Ke:nx para ordenador Macintosh, distribuido por Don Johnston; el DADA para ordenador tipo PC, distribuido por TASH.

\section{Formas de acceso}

Son todos aquellos instrumentos que facilitan que personas con graves dificultades de indicación directa puedan acceder más fácilmente a comunicadores, ordenadores, etc. Cabe destacar los siguientes:

- Cabezal licornio. Consiste en una varilla que se acopla a la cabeza. Puede encontrarse en las tiendas de ortopedia.

- Lápiz óptico. El usuario señala con un tubo de tamaño de un lápiz colocado en una parte del cuerpo, normalmente la cabeza, un tablero situado en la pantalla, con los mismos caracteres que el teclado, después de un tiempo predeterminado, se activa el carácter señalado. Distribuido por Pointer Systems, Inc.

- Conmutadores. Todos tienen en común que el usuario es capaz, abriendo y cerrando un circuito eléctrico, de enviar una señal al ordenador, comunicador, juguete adaptado, etc. Puden ser de tipos y tamaños muy variados, adaptándose a las posibilidades motoras del usuario. Distribuidos, entre otros, por AbbleNet, Don Johnston, QED, TASH, etcétera.

Sintetizadores de voz. Periférico de salida muy útil cuando se utiliza el ordenador como ayuda técnica para la comunicación. Ultimamente se están haciendo grandes avances en este campo y actualmente podemos contar con los siguientes:

- CIBERVOZ-CIBERVEU. Interfase conversor texto-voz, cuenta con una tarjeta o una caja autónoma. Disponible para ordenadores compatibles PC. Dispone de voz en castellano y en catalán, pero no es portátil.

- INFOVOX. Existe en diferentes idiomas entre ellos el castellano. Cuenta con diversas versiones portátiles. Disponible para compatibles PC. Distribuido por Comelta.

- APOLO. Existe en diferentes idiomas entre ellos el castellano. Existen diversas versiones portátiles. Disponible para compatibles PC. Distribuido en España por Canon. 


\section{Software}

Existen programas de ordenador para ser utilizados con los diferentes periféricos nombrados y de distintos niveles, pudiéndose adaptar a las características y necesidades del usuario. Podemos catalogarlos en grandes bloques temáticos:

- Programas de causa-efecto, adecuados para usuarios con un nivel cognitivo bajo. También permiten valorar el uso de conmutadores.

- Aprendizaje de conceptos básicos, como tamaños, colores, formas, clasificaciones, etc.

- Dibujo, permiten que usuarios de conmutadores u orros periféricos puedan realizar diferentes actividades gráficas.

- Cálculo, desde conceptos básicos de cantidad a operaciones matemáticas complejas.

- Comunicación y lenguaje escrito. Existen diferentes programas de comunicación mediante signos gráficos y escritura tradicional, que pueden ser utilizados con voz sintetizada y mediante diferentes formas de acceso.

Son indicados los programas que permiten un fácil acceso al terapeuta, pudiendo variar rutinas y permitiendo una mayor adaptación.

Hay que tener en cuenta la compatibilidad entre los diferentes ordenadores en el mercado, en el momento de realizar la selección. Puede encóntrarse software en las casas comerciales de ordenadores y en los centros de recursos educativos, también cuentan con software especial: ACE Centre, ACS, Don Johnston, Edicinco, Mayer Johnson, etcétera.

\section{Juguetes adaptados}

Permiten a niños con poco control motor poder activar juguetes que, de otra forma, no podrían manipular. Mediante una sencilla adaptación, juguetes u otros aparatos (casetes, luces, etc.), con pilas o conectados a la corriente eléctrica, pueden ser controlados mediante conmutadores, adaptándose a las posibilidades motoras y cognitivas del niño.

Algunas casas comercializan juguetes especialmente diseñados para niños con pluridiscapacidad. Son distribuidores: AbbleNet y Toys for Special Children.

\section{ENTIDADES}

Seguidamente se exponen las principales entidades que pueden proporcionar información en relación a la comunicación aumentativa y ayudas técnicas. Se ha realizado una selección a nivel estatal, exceptuando la ISAAC (Sociedad Internacional para la Comunicación Aumentativa y Alternativa) por su especial significación en este campo.

ISAAC. International Society for Augmentative and Alternative Communication. Dirigir la correspondencia en inglés a Liz Baer: P.O. Box 1762, Station $R$, Toronto, Ontario, M4G 4A3. CANADA. Correspondencia en castellano a Carme Basil: Departament de Psicologia Evolutiva i de l'Educació. Universitat de Barcelona. Adolf Florensa s/n. 08028 BARCELONA. Tel.: (93) 3333466 , ext. 3564.

Sociedad internacional dedicada a la investigación y divulgación de temas relacionados con la Comunicación Aumentativa y Alternativa. Su órgano oficial es la revista AAC, Augmentative and Alternative Communication. 
Asociación Española de Logopedia, Foniatria y Audiología. Violant dHungria, 11-115, esc. B, pral. 4." O8028 BARCELONA. Tel.: (93) 3309141.

Su órgano oficial es la Revista de Logopedia, Foniatría y Audiología.

Centro Nacional de Recursos para la Educación Especial (C.N.R.E.E.). MINISTERIO DE EDUCACION Y CIENCIA. General Oráa, 55. 28006 MADRID. Tels.: (91) $5621190-5620608$.

Contro der endiente del Ministerio de Educación y Ciencia, donde se cuenta con material especifico, publicaciones, etc., referidas a las necesidades educativas especiales de niños coti ficiencia motriz.

Centro Estatal de Autonomía Personal y Ayudas Técnicas (C.E.A.P.A.T.). Los Extremeños, 1. 28038 MADRID. Teléfono: 34-1-7789061. Fax: 34-1-77841 17.

Cencro dependiente del Instituto Nacional de Servicios Sociales INSERSO. Cuentan con material específico y ayudas técnicas para el desplazamiento, habilitación y comunicación de personas con discapacidad.

Departament D'ensenyament Generalitat de Catalunya. Diagonal, 682. 08034 BARCELONA. Tel.: (93) 2051000.

Publican la colección Documents d'Educació Especial.

Fundesco. Fundación para el Desarrollo de la Función Social de las Comunicaciones. Alcalá, 61. 28014 MADRID. Tel: (91) 4351214.

Publican juntamente con el Real Patronato: Los Libros de FUNDESCO, con la Colección Estudios y Documentos.

O.N.C.E. Unidad Tiflotécnica. Ramírez de Arellano, 21, 28043 MADRID. Teléfono: 4150600 .

Información sobre diferentes ayudas técnicas para personas con déficits visuales: pantallas ampliadas para ordenador, etcétera.

P.I.E. Programa Informática Educativa. Via Laietana, 64, $1 . B A R C E L O-$ NA. Tels.: (93) $4120603-4121962$. Fax: 3011345.

Realizan y distribuyen programas de ordenador y material informático a las escuelas dependientes de la Generalitat de Catalunya.

Pont del Drago. Andreu Nin, s/n.08016 BARCELONA .Tel.: (93) 3536447.

Centro ocupacional y de formación profesional ocupacional para jóvenes con grandes disminuciones físicas, cuenta con ayudas técnicas para la comunicación y ocupación. Dependiente del Institut Municipal de Disminuïts del Ayuntamiento de Barcelona.

Real Patronato de Prevención y de Atención a Personas con Minusvalía. Serrano, 140. 28006 MADRID. Tel.: (91) 2627337.

Editan un boletín que informa sobre legislación, noticias relacionadas, jornadas, etc. Cuenta con un apartado de documentación sobre libros, bibliografía, vídeos y publicaciones del Real Patronato.

SIRIUS. Centre de Referencia per a Disminuits. Calabria, 129-131. 08015 BARCELONA. Tel.: (93) 48413 O0. Fax: (93) 4262877.

Centro dependiente del Departament de Benestar Social de la Generalitat de Catalunya. Ofrece información, orientación y asesoramiento a profesionales, entidades públicas y privadas, hospitales, escuelas, etc., relacionadas con las personas con discapacidad. Dispone de exposición de ayudas técnicas.

UTAC-NADIS. Canaries, 1.08017 BARCELONA. Tel.: (93) 4173679.

Servicio de atención e información sobre técnicas aumentativas de comunicación, dirigido a usuarios, familiares y profesionales. Concertado con el Departament de Benestar Social de la Generalitat de Catalunya. 


\section{DIRECCIONES DE INTERES}

En este apartado se relacionan algunas casas comerciales, que proporcionan catálogos, para que las personas directamente relacionadas con la comunicación aumentativa y las ayudas técnicas puedan estar informadas de los instrumentos útiles para ello que aparecen en el mercado.

AB MICRO EXPRESS. Wharfdale Road, CF2 7HB, Pentwyn, Cardiff, G.B. Tel.: 0222733485.

ABBLENET. Inc. 1081 Tenth Avenue S.E. Minneapolis MN 55414-1312, EE.UU. Tel.: 612 3790956. Fax: 612-379-9143.

ACS. ADAPTIVE COMMUNICATION SYSTEMS, Inc. Box 12440, PA15231 Pittsburgh, EE.UU. Tel.: 412 262-2288.

CANON Ltd. Personal Products Division, Canon House, Manor Rd, Wallington, SM6 OAL, Surrey, G.B. Tel.: 081773 3173. Fax: 08177321 56. CANON ESPAÑA. Joaquín Costa, 41.28002 Madrid. Tel.: (91) 5384513.

CIBERVOZ- CIBERVEU. Aribau, 162-166, Entr. J. 08036 BARCELONA. Tels.: (93) 2382648 2382771 . Fax: 4152594.

COMELTA. Pedro IV, 84, 5.008005 BARCELONA. Tel.: (93) 30077 12. Telefax: 3433005156. Télex: 51934 CETEA E. Distribuidores de INFOVOX: P.O. Box 2503, S-17102 Solna (Suecia). Tel.: 46873580 90. Fax: 4687357876 .

DON JOHNSTON, DEVELOPMENTAL EQUIPMENT, Inc. P.O. Box 639, 1000 N. Rand Rd., Bldg. 115, IL, Wauconda, EE.UU. Tel.: 708-526-2682. Fax: 708 526-4177.

EDICINCO. Plátanos, 30-32. 46025 VALENCIA. Tel.: (96) 34847 28. Software para ser usado con conmutadores, para ordenadores comparibles P.C.

FONDATION SUISSE POUR LES TELETHESES. Cret-Taconnet 32, Case postale 1755, CH2002 Neuchatel, Suiza. Tel.: 038246757.

LIBERATOR Ltd. PRENTKE ROMICH EUROPE. Whitegates, Swinstead, Lincs NG33 4PA. G.B. Tel.: 047684391 . Fax: 047684357.

MARDIS. Room 22, Enginnering Building, Lancaster University, Bailrigg, Lancaster LA1 4VW. G.B. Tel.: 052465201 .Ext. 3692.

MAYER JOHNSON P.O. Box 1579, Solana, CA 92075-1579, EE.UU. Tel.: 619481 2489. Fax: 619 2595726.

MULTITEC. Félix Pizcueta, 10. 46004 VALENCIA. Tel.: (96) 35147 27. Fax: (96) 3255134.

PERSONIC CORPORATION. 2352 Main Street, Concord, MA 017792, EE.UU. Tel.: 617897 1575.

POINTER SYSTEMS Inc. One Mill Street, Burlington, Vermont 05401, EE.UU. Tel.: 8026583260. Fax: 8026583714.

POSSUM CONTROLS Lrd. Middlegreen Road, Langley, Slough, Berks, SL3 6DF. G.B. Tel.: 0753 79234. Fax: 0753512840.

P.R.C. PRENTKE ROMICH COMPANY. 211022 Heyl Road, Wooster, 44691 Ohio, EE.UU. Tel.: 2162621984 . Fax: 2162634829.

QUEST EDUCATIONAL DESINGS Ltd. Prince Alfred Street, Gosport, Hants PO12 1QH. G.B. Tel.: 0705581178.

TASH. TECHNICAL AIDS \& SYSTEMS FOR THE HANDICAPPED Inc. 70 Gibson Dr., Unit 12, Markham, Ontario L3R 4C2, Canadá. Tel.: 416475 2212. Fax: 4164752422.

TOYS FOR SPECIAL CHILDREN 358 Warburton Ave., Hastings on Hudson, NY 10706, EE.UU. Tel.: 9144780960 . Fax: 9144787030.

ZYGO INDUSTRIES, Inc. PO BOX 1008. Portland, Oregon 97207-1008. EE.UU. Comercializado en nuestro país por: MULTITEC. Maestro Rodrigo, 103 bajo. 46015 VALENCIA. Tel.: (93) 34725 86. Fax: (96) 3471179. 